Interfaces and Free Boundaries 13 (2011), 171-189

DOI $10.4171 / \mathrm{IFB} / 253$

\title{
Local simplicity, topology, and sets of finite perimeter
}

\author{
David G. CARABALlo \\ Department of Mathematics, Georgetown University, \\ St. Mary's Hall, 3rd floor, Washington, D.C. 20057-1233, USA \\ E-mail:dgc3@georgetown.edu
}

[Received 29 August 2009 and in revised form 28 October 2010]

\begin{abstract}
We introduce a useful and relatively easy to check condition, local simplicity, which provides significantly more structure to sets of finite perimeter, while not being too restrictive. Local simplicity holds for minimizers in a wide variety of variational problems in materials science, biology, image processing, oncology, and other fields. We prove several regularity and structural properties of locally simple sets and their boundaries, including a vital decomposition theorem that in our setting strengthens the conclusion of theorems of H. Federer and L. Ambrosio, V. Caselles, S. Masnou, and J.-M. Morel. We establish strong connections between topology and sets of finite perimeter, so that ordinary notions of openness, closedness, and connectedness may be readily used in the finite perimeter setting.

We apply these results to an image reconstruction procedure from image processing, $L^{1}$ TVminimization. The density ratio bounds computed to establish local simplicity are themselves of practical importance, as they provide concrete, easy to compute criteria to check simulations against.
\end{abstract}

2010 Mathematics Subject Classification: 49N60, 49Q20, 49M27, 94A08, $68 \mathrm{U} 10$.

Keywords: Local simplicity; decomposable; finite perimeter; regularity; reduced boundary; measuretheoretic boundary; total variation; TV-minimization.

\section{Introduction}

Sets of locally finite perimeter, those whose characteristic functions have locally bounded variation, have become a natural setting in which to study many problems involving surfaces and interfaces, in areas such as materials science, fluid mechanics, surface physics, image processing, oncology, and computer vision (see, for example, [1], [2], [3], [5]-[9], [11], [12], [14]-[20], [23], [27], [28], [30], [31], [33], [38]-[40], and the many references cited therein). They are general enough to adequately model complex physical phenomena with singularities, they have useful local approximation properties, and they satisfy vital compactness results which do not hold for classes of sets having smooth boundaries.

Working in the context of sets of finite perimeter, while often desirable, introduces various technical difficulties as a consequence of their general nature. Their reduced boundaries may contain elaborate filigree structures even to the extent that the boundary closures have infinite volume (see Example 9. Typically in sharp interface models we would at the very least like the boundary closures to be $(n-1)$-dimensional.

Also, in a fundamental sense the measure-theoretic and topological aspects of a set are incompatible. Measure-theoretic aspects of a set, such as its reduced boundary, are generally unaltered if we make changes on a set having $\mathcal{L}^{n}$ measure zero. At the other extreme, whether or not a set is open, closed, or connected can depend significantly on a single point, and so substantial 
care needs to be taken if we are to try to use these and other topological ideas when working in the finite perimeter setting from geometric measure theory.

Yet, these topological concepts are vital for theoretical and computational analysis in various fields. Indeed, the importance of connectedness in image processing was a strong motivating factor for L. Ambrosio, V. Caselles, S. Masnou, and J.-M. Morel. In their paper [8], they specialized H. Federer's notion of indecomposable integral currents ([25, 4.2.25])-the measure-theoretic analogues of connected sets- to the context of sets of finite perimeter. In that setting they established results analogous to the usual topological theorems concerning connectedness, so that topology-like connectedness arguments could be imitated in the finite perimeter setting, and so that operations in image processing that used connectedness in that setting could be rigorously studied and justified.

Of particular importance is their Decomposition Theorem ([8, Theorem 1]), the specialization of $([25,4.2 .25])$ to the finite perimeter setting, in which they use a new argument to show that any set of finite perimeter may be uniquely decomposed as a countable union of disjoint, maximal indecomposable sets in such a way that the perimeter of the set is the sum of the perimeters of its indecomposable parts.

Our primary motivation was to find somewhat unrestrictive conditions under which sets of finite perimeter would be measure-theoretically and topologically compatible, in order to help with theoretical analysis, strengthen and justify computational analysis, and generally facilitate the use of sets of finite perimeter in applications.

Since sets of finite perimeter are essentially defined only up to a set having $\mathcal{L}^{n}$ measure zero, we will pick specific representatives from each class by choosing those points with $n$-dimensional density equal to one. Thus, we will often work with $\operatorname{Int}(K)=\left\{x \in \mathbb{R}^{n}: \Theta^{n}(K, x)=1\right\}$ instead of $K$, and with $\operatorname{Ext}(K)=\operatorname{Int}\left(\mathbb{R}^{n} \backslash K\right)$ instead of $\mathbb{R}^{n} \backslash K$. Practically speaking, there is no loss of generality in doing this since $K$ and $\operatorname{Int}(K)$ differ at most on a set having $\mathcal{L}^{n}$ measure 0 , and similarly for $\mathbb{R}^{n} \backslash K$ and $\operatorname{Ext}(K)$. Also, they all have the same reduced boundaries and the same measure-theoretic boundaries (see Theorem 2).

Still, $\operatorname{Int}(K)$ and $\operatorname{Ext}(K)$ can be quite complicated objects. The key condition which strongly links their topological and measure-theoretic properties seems to be local simplicity, defined in Section 3 Local simplicity (see Definition 4 4 is general enough to hold for a wide variety of variational problems involving sets and their boundaries. It allows us to establish strong connections between topology and sets of finite perimeter. Many topological results and constructions that are desirable for applications, but which do not hold for general sets of finite perimeter, may be employed in both theoretical and computational analyses once we verify that local simplicity holds for a given application.

The defining condition for local simplicity involves continuous lower and upper bounds to $n$ dimensional density ratios to keep the densities away from 0 and 1 . Such conditions (with constant bounds) have been established for a variety of minimization problems (see, for example, [5], [10], [17], [30], and [40]), generally as an intermediate step in trying to establish additional structural and regularity results.

Example 14 gives some indication of how important local simplicity is if we want the measuretheoretic and topological boundaries of $\operatorname{Int}(K)$ to be the same. In that example, the $n$-dimensional density fails to stay away from 0 and 1 at just a single point, and $\partial_{\text {top }}(\operatorname{Int}(K)) \neq \partial^{M}(\operatorname{Int}(K))$.

We establish several partial regularity and structural results for locally simple sets (Theorems 7. 8, and 10, and Proposition 15. We also show that, in the setting of locally simple sets, we get 
agreement among several different notions of boundary, both measure-theoretic and topological (Theorem 11 and Remark 12).

We give fundamental results concerning measure-theoretic and topological notions of connectedness (Theorem 17 and Proposition [18). We also establish a decomposition theorem (Theorem 6), expressing measure-theoretic interiors and exteriors of locally simple sets uniquely as countable unions of disjoint, open connected sets, and we show that the perimeter of the set is the sum of the perimeters of the components. We also show that, for locally simple sets, our decomposition agrees exactly with that of [8, Theorem 1]. Our result therefore allows us to make use of theorems and constructions from [8] as well.

Previously, H. Federer ([25, 4.2.25]) and L. Ambrosio, V. Caselles, S. Masnou, and J.-M. Morel ([8]) had established related results, decomposing an arbitrary set of finite perimeter into pairwise disjoint, maximal indecomposable parts.

Finally, as an example application we consider the method of $L^{1} \mathrm{TV}$-minimization, a variational method for image reconstruction related to the popular ROF model of L. Rudin, S. Osher, and E. Fatemi [35]. $L^{1}$ TV-minimization is a well-known method, first considered in a discrete setting by S. Alliney and M. Nikolova (see, for example, [4] and [34]), and later in a continuous setting for $n \geqslant 2$ by T. Chan and S. Esedoglu [20], W. Allard ([1], [2], [3]), and others.

We show that, under reasonable and quite general conditions, super-level sets $E_{y}=\{u>y\}$ associated with $L^{1}$ TV-minimizers $u$ are locally simple (Theorems 23 and 25. These results imply that $E_{y}$ has each of the regularity and structural properties given in Sections 3 and 4

We proceed by deriving explicit continuous lower and upper bounds for the $n$-dimensional density ratios $\Theta^{n}\left(E_{y}, x, r\right)$ at points $x \in \overline{\partial E_{y}}$. These computed bounds are also of practical value, since they can be used to try to eliminate some noise or uncertainty in the images and since they can also be used to test computer implementations of the $L^{1} \mathrm{TV}$-minimization procedure.

\section{Notation and BV functions}

We will measure volume and surface area in $\mathbb{R}^{n}$ (for $n \geqslant 2$ ) with $n$-dimensional Lebesgue measure $\mathcal{L}^{n}$ and $(n-1)$-dimensional Hausdorff measure $\mathcal{H}^{n-1}$, respectively. We let $B(p, r)$ and $U(p, r)$ denote, respectively, the closed and open balls in $\mathbb{R}^{n}$ with center $p$ and radius $r$, and we set $\alpha(n)=\mathcal{L}^{n}(B(\mathbf{0}, 1))$, where $\mathbf{0}=(0, \ldots, 0)$ is the origin in $\mathbb{R}^{n}$. If $A, B \subset \mathbb{R}^{n}$ and $0 \leqslant m \leqslant n$, we write $A \subset_{m} B$ (i.e., " $A$ is $\mathcal{H}^{m}$ almost contained in $B$ ") when $\mathcal{H}^{m}(A \backslash B)=0$. If $A, B \subset \mathbb{R}^{n}, A \triangle B$ denotes the symmetric difference of $A$ and $B: A \triangle B=(A \backslash B) \cup(B \backslash A)$; also, we write $A={ }_{m} B$ provided $\mathcal{H}^{m}(A \triangle B)=0$. If $A \subset \mathbb{R}^{n}$ is $\mathcal{L}^{n}$ measurable, we let $\chi_{A}$ denote the characteristic function of $A$, taking the value 1 inside $A$ and the value 0 outside $A$.

Given a point $p \in \mathbb{R}^{n}$ and a unit vector $u \in \mathbb{R}^{n}$, we define the open half-spaces $H_{+}(p, u)=$ $\{x:(x-p) \cdot u>0\}$ and $H_{-}(p, u)=\{x:(x-p) \cdot u<0\}$. We call a set countable if it is finite or countably infinite.

If $A \subset \mathbb{R}^{n}, \bar{A}$ denotes the closure of $A$ in $\mathbb{R}^{n}$. When $A \subset \mathbb{R}^{n}$, we let $\partial_{\text {top }} A=\bar{A} \cap \overline{\mathbb{R}^{n} \backslash A}$ denote the topological boundary of $A$. We note that $x \in \partial_{\text {top }} A$ if and only if for each $r>0$ we have $U(x, r) \cap A \neq \emptyset$ and $U(x, r) \cap\left(\mathbb{R}^{n} \backslash A\right) \neq \emptyset$.

If $X \subset \mathbb{R}^{n}$ is $\mathcal{H}^{m}$ measurable $(1 \leqslant m \leqslant n)$ and $p \in \mathbb{R}^{n}$, the $m$-dimensional density of $X$ at $p$ is

$$
\Theta^{m}(X, p)=\lim _{R \rightarrow 0^{+}} \frac{\mathcal{H}^{m}(X \cap B(p, R))}{\alpha(m) R^{m}},
$$

provided the limit exists. When $X \subset \mathbb{R}^{n}$ is $\mathcal{L}^{n}$ measurable, $p \in \mathbb{R}^{n}$, and $R>0$, we define the 
$n$-dimensional density ratio of $X$ at $p$ in $B(p, R)$ as

$$
\Theta^{n}(X, p, R)=\mathcal{L}^{n}(X \cap B(p, R)) / \mathcal{L}^{n}(B(p, R)),
$$

and we observe that $\Theta^{n}(X, p)=\lim _{R \rightarrow 0^{+}} \Theta^{n}(X, p, R)$, provided the limit exists.

If $X \subset \mathbb{R}^{n}$ is $\mathcal{L}^{n}$ measurable and $0 \leqslant d \leqslant 1$, we let $X^{d}=\left\{p \in \mathbb{R}^{n}: \Theta^{n}(X, p)=d\right\}$ denote the set of points at which $X$ has density $d$, and we let $\operatorname{Int}(X)=X^{1}$ and $\operatorname{Ext}(X)=X^{0}$ denote the measure-theoretic interior and measure-theoretic exterior of $X$, respectively. We let $\partial^{M} X=$ $\mathbb{R}^{n} \backslash(\operatorname{Int}(X) \cup \operatorname{Ext}(X))$ denote the measure-theoretic boundary of $X$.

If $X \subset \mathbb{R}^{n}$ and $p \in \mathbb{R}^{n}$, the vector $u \in \mathbb{R}^{n}$ is called a measure-theoretic exterior unit normal to $X$ at $p$ in the sense of Federer (cf. [24], [25, 4.5.5]) provided $|u|=1, \Theta^{n}\left(H_{+}(p, u) \cap X, p\right)=0$, and $\Theta^{n}\left(H_{-}(p, u) \backslash X, p\right)=0$. If no such $u$ exists, we define $n_{X}(p)=\mathbf{0}$, while if such a $u$ exists it is necessarily unique ([24, Theorem 3.4]) and we define $n_{X}(p)=u$.

Whenever $K$ is an $\mathcal{L}^{n}$ measurable subset of $\mathbb{R}^{n}$ we let $\partial K$ denote the reduced boundary of $K$ (see [21]-[23]) in the sense of De Giorgi. Whenever $K$ is $\mathcal{L}^{n}$ measurable and $p \in \partial K, n_{K}(p)$ is a unit vector. If $K$ and $L$ are $\mathcal{L}^{n}$ measurable subsets of $\mathbb{R}^{n}$ with $\mathcal{L}^{n}(K \triangle L)=0$, then $\partial K=\partial L$.

Whenever $K \subset \mathbb{R}^{n}$ is $\mathcal{L}^{n}$ measurable, $p \in \partial K$, and $R>0$,

$$
\Theta^{n-1}(\partial K, p, R)=\frac{\mathcal{H}^{n-1}(\partial K \cap B(p, R))}{\alpha(n-1) R^{n-1}}
$$

is the $(n-1)$-dimensional density ratio of $\partial K$ at $p$ in $B(p, R)$.

Throughout the paper, $\Omega$ will denote an open subset of $\mathbb{R}^{n}$. Let $u \in L_{\text {loc }}^{1}(\Omega)$. Whenever $U \subset \Omega$ is open, we define the total variation of $u$ in $U$ as follows:

$$
\operatorname{TV}(u, U)=\sup _{\substack{\phi \in C_{c}^{1}\left(U, \mathbb{R}^{n}\right) \\|\phi(x)| \leqslant 1 \text { for all } x \in U}}\left\{\int_{x \in U} u(x) \operatorname{div} \phi(x) \mathrm{d} \mathcal{L}^{n} x\right\}
$$

We can extend $\operatorname{TV}(u, \cdot)$ to be a Borel measure on $\Omega$ by setting

$$
\operatorname{TV}(u, E)=\inf \{\operatorname{TV}(u, U): U \text { is an open set containing } E\}
$$

for any Borel set $E$ in $\Omega$. Then $\operatorname{TV}(u, \cdot)$ is a Radon measure on $\Omega$ if and only if $\operatorname{TV}(u, K)<\infty$ for all compact $K \subset \Omega$. We note that, if $u \in C^{1}(\Omega)$, then integration by parts gives $\operatorname{TV}(u, \Omega)=$ $\int_{\Omega}|\nabla u| \mathrm{d} \mathcal{L}^{n}$

We let

$$
\mathrm{BV}(\Omega)=\left\{f: f \in L^{1}(\Omega), \text { and } \operatorname{TV}(f, \Omega)<\infty\right\}
$$

denote the space of functions of bounded variation in $\Omega$, and we let

$$
\operatorname{BV}_{\text {loc }}(\Omega)=\left\{f: f \in L_{\text {loc }}^{1}(\Omega), \text { and } \operatorname{TV}(f, K)<\infty \text { for all compact } K \subset \Omega\right\}
$$

be the space of functions of locally bounded variation in $\Omega$.

When $A$ is an $\mathcal{L}^{n}$ measurable subset of $\mathbb{R}^{n}$, we let $P(A, \Omega)=\operatorname{TV}\left(\chi_{A}, \Omega\right)$ be the perimeter of $A$ in $\Omega$; also, we say that $A$ has finite perimeter in $\Omega$ provided $P(A, \Omega)<\infty$, and we say that $A$ has locally finite perimeter in $\Omega$ provided $\chi_{A} \in \operatorname{BV}_{\text {loc }}(\Omega)$. When $\Omega=\mathbb{R}^{n}$, we write $P(A)=P\left(A, \mathbb{R}^{n}\right)$, and we let $\mathcal{P}$ denote the collection of all $\mathcal{L}^{n}$ measurable sets $A \subset \mathbb{R}^{n}$ having finite perimeter in $\mathbb{R}^{n}$. We have $P(A)=\mathcal{H}^{n-1}(\partial A)<\infty$ whenever $A \in \mathcal{P}$ (see [22]). 
If $A \subset \mathbb{R}^{n}$ is Lebesgue measurable and has locally finite perimeter in $\mathbb{R}^{n}$, we let $[A]$ be the multiplicity one integral $n$-current associated with $A$, defined (cf. [7, 3.1.4], [1, Definition 2.1]) according to

$$
[A](w)=\int_{x \in A}\left\langle e_{1} \wedge \cdots \wedge e_{n}, w(x)\right\rangle \mathrm{d} \mathcal{L}^{n} x
$$

for each $C^{\infty}$ differential $n$-form $w$ with compact support. We will not discuss integral currents here in detail—see [25], [33], [29], or [7] for more details.

$\operatorname{spt}[A]$ is the smallest closed set $Q$ such that

$$
(\text { spt } w) \cap Q=\emptyset \Rightarrow[A](w)=0
$$

whenever $w$ is a smooth differential $n$-form with compact support ([25, 4.1.1], [33, 4.2]). For convenience we state and prove the following elementary result, which is used several times in this paper.

PROPOSITION 1 Suppose $K$ is a Lebesgue measurable subset of $\mathbb{R}^{n}$ having locally finite perimeter. Then $\overline{\partial K} \subset \operatorname{spt}[K]$.

Proof. Suppose $x \in \partial K \backslash \operatorname{spt}[K]$, and let $r>0$ be such that $U(x, r) \cap \operatorname{spt}[K]=\emptyset$. We can find $r^{\prime} \in(0, r)$ so that $U\left(x, r^{\prime}\right) \cap K$ is very nearly a half-ball, and we can then easily construct a $C^{\infty}$ differential $n$-form $w$, with compact support contained inside $B\left(x, r^{\prime}\right)$, for which $[K](w)>0$, contradicting the definition of spt $[K]$ as given in (3). Therefore $\partial K \subset \operatorname{spt}[K]$ and the result follows by taking closures.

BV functions are intermediate between Sobolev functions and $L^{1}$ functions. Specifically, we have the inclusions

$$
W_{\mathrm{loc}}^{1,1}(\Omega) \subset \mathrm{BV}_{\mathrm{loc}}(\Omega) \subset L_{\mathrm{loc}}^{1}(\Omega) \quad \text { and } \quad W^{1,1}(\Omega) \subset \mathrm{BV}(\Omega) \subset L^{1}(\Omega) .
$$

BV functions are more suitable than Sobolev functions for many image processing and materials science applications since, for instance, they include characteristic functions of fairly general subsets of $\mathbb{R}^{n}$. For example, if $K$ is a bounded, $\mathcal{L}^{n}$ measurable subset of $\mathbb{R}^{n}$, then $\chi_{K} \in \mathrm{BV}\left(\mathbb{R}^{n}\right)$ if and only if $K \in \mathcal{P}$. By contrast, $W^{1,1}(\Omega)$ does not contain characteristic functions of bounded, $\mathcal{L}^{n}$ measurable subsets of $\mathbb{R}^{n}$ having $C^{2}$ boundary (see [26, 1.4]). Also, unlike $W^{1,1}(\Omega)$, spaces of BV functions have useful compactness properties essential for variational problems (cf. [9, Theorem 3.23]). Some excellent references that treat sets of finite perimeter and functions of bounded variation in detail are [1], [9], [15], [23], [26], [28], and [31].

It is often preferable to work with $\operatorname{Int}(K)$ and $\operatorname{Ext}(K)$ instead of $K$ and $\mathbb{R}^{n} \backslash K$. Indeed, Example 5 shows that the topological boundary of $K$ can be quite difficult to control; yet, we will find (see Theorem 111) that the topological boundaries of $\operatorname{Int}(K)$ and $\operatorname{Ext}(K)$ agree with their measure-theoretic boundaries when $K$ is locally simple. Theorem 2 shows that we may work with $\operatorname{Int}(K)$ and $\operatorname{Ext}(K)$ instead of $K$ and $\mathbb{R}^{n} \backslash K$ without loss of generality.

THEOREM 2 (On interiors, exteriors, and boundaries) Suppose $K \in \mathcal{P}$. Then

(i) $\mathcal{L}^{n}(K \triangle \operatorname{Int}(K))=0$.

(ii) $\mathcal{L}^{n}\left(\left(\mathbb{R}^{n} \backslash K\right) \triangle \operatorname{Ext}(K)\right)=0$.

(iii) $\partial(\operatorname{Int}(K))=\partial K=\partial\left(\mathbb{R}^{n} \backslash K\right)=\partial(\operatorname{Ext}(K))$, and in particular

$$
P(\operatorname{Int}(K))=P(K)=P\left(\mathbb{R}^{n} \backslash K\right)=P(\operatorname{Ext}(K)) .
$$


(iv) $\partial^{M}(\operatorname{Int}(K))=\partial^{M} K=\partial^{M}\left(\mathbb{R}^{n} \backslash K\right)=\partial^{M}(\operatorname{Ext}(K))$.

(v) $\partial K \subset K^{1 / 2} \subset \partial^{M} K$.

(vi) $\mathcal{H}^{n-1}\left(\partial^{M} K \backslash \partial K\right)=0$ and $\partial K={ }_{n-1} K^{1 / 2}={ }_{n-1} \partial^{M} K$.

(vii) $\mathcal{H}^{n-1}\left(\mathbb{R}^{n} \backslash(\operatorname{Int}(K) \cup \partial K \cup \operatorname{Ext}(K))\right)=0$.

Proof. The Lebesgue Differentiation Theorem implies that $\mathcal{L}^{n}(K \backslash \operatorname{Int}(K))=0$, and [25, 2.9.11] shows that $\mathcal{L}^{n}(\operatorname{Int}(K) \backslash K)=0$ if and only if $K$ is $\mathcal{L}^{n}$ measurable. Since $K$ is $\mathcal{L}^{n}$ measurable, (i) holds. (ii) follows by applying the same argument to $\mathbb{R}^{n} \backslash K$ instead of $K$. That $\partial K=\partial\left(\mathbb{R}^{n} \backslash K\right)$ follows immediately from the definitions (see [25, 4.5.5] and [25, 4.5.6]). The first and third equalities in (iii) follow from (i) and (ii), respectively. The equalities for perimeter follow from these results and from [26, Remark 1.7(iii)]. Next, we compute

$$
\partial^{M}\left(\mathbb{R}^{n} \backslash K\right)=\mathbb{R}^{n} \backslash\left(\operatorname{Int}\left(\mathbb{R}^{n} \backslash K\right) \cup \operatorname{Ext}\left(\mathbb{R}^{n} \backslash K\right)\right)=\mathbb{R}^{n} \backslash(\operatorname{Ext}(K) \cup \operatorname{Int}(K))=\partial^{M} K .
$$

Whenever $X, Y \in \mathcal{P}$ and $\mathcal{L}^{n}(X \triangle Y)=0$, we can conclude that $\operatorname{Int}(Y)=\operatorname{Int}(X)$ and $\operatorname{Ext}(Y)=$ $\operatorname{Ext}(X)$ since $\mathcal{L}^{n}(Y \cap U(x, R))=\mathcal{L}^{n}(X \cap U(x, R))$ for each $R$. Letting $X=\operatorname{Int}(K)$ and $Y=K$, we have $\operatorname{Int}(X)=\operatorname{Int}(K)$, and $\operatorname{Ext}(X)=\operatorname{Ext}(K)$, so that

$$
\partial^{M} X=\mathbb{R}^{n} \backslash(\operatorname{Int}(X) \cup \operatorname{Ext}(X))=\mathbb{R}^{n} \backslash(\operatorname{Int}(K) \cup \operatorname{Ext}(K))=\partial^{M} K .
$$

We obtain $\partial^{M}(\operatorname{Ext}(K))=\partial^{M}\left(\mathbb{R}^{n} \backslash K\right)$ similarly, and (iv) follows.

If $p \in \partial K$, then $K$ has a measure-theoretic exterior unit normal, $n_{K}(p)$, satisfying $\Theta^{n}\left(H_{+}\left(p, n_{K}(p)\right) \cap K, p\right)=0$ and $\Theta^{n}\left(H_{-}\left(p, n_{K}(p)\right) \backslash K, p\right)=0([25,4.5 .5])$, so $\Theta^{n}(K, p)=$ $\Theta^{n}\left(\mathbb{R}^{n} \backslash K, p\right)=1 / 2$, and thus $p \in K^{1 / 2}$. Any $p \in K^{1 / 2}$ has density $1 / 2$, hence not 0 or 1 , so the second inclusion in (v) follows. The assertion that $\mathcal{H}^{n-1}\left(\partial^{M} K \backslash \partial K\right)=0$ is simply [23, Lemma 1(ii)]. The second statement of (vi) follows from the first and from (v); and (vii) follows from 25 , 4.5.6].

We will make use of the following standard result several times throughout this paper (see, for example, [28, Corollary 3.7.14]).

THEOREM 3 (Relative isoperimetric inequality) There exists a constant $C(n)$, depending only on $n$, with the following property. Suppose $A$ is an $\mathcal{L}^{n}$ measurable subset of $\mathbb{R}^{n}$ having locally finite perimeter. Suppose $U$ is any open ball in $\mathbb{R}^{n}$. Then

$$
\min \left\{\mathcal{L}^{n}(A \cap U), \mathcal{L}^{n}\left(\left(\mathbb{R}^{n} \backslash A\right) \cap U\right)\right\}^{(n-1) / n} \leqslant C(n) P(A, U) .
$$

\section{Local simplicity}

The following density ratio condition will be indispensable in precluding pathological boundary behavior and in helping to establish additional regularity for boundaries of sets having locally finite perimeter. It is frequently satisfied by minimizers to problems involving perimeter. See, for example, [5], [7], [10], [16], [17], [26], [30], [33], and [40] for the general method of establishing density ratio conditions. Typically, the conditions in the literature involve the functions $a(x), A(x)$, and $\delta(x)$ from Definition 4 being constant, so that the density ratio bounds are uniform. We establish our results in the more general setting of continuous density ratio bounds, a setting which is more suitable if, for instance, the sets are unbounded. 
Definition 4 A set $K \in \mathcal{P}$ is called locally simple, and we write $K \in \mathcal{P}_{\mathrm{LS}}$, provided $\partial K \neq \emptyset$ and there exist continuous functions $\delta, a, A: \mathbb{R}^{n} \rightarrow[0, \infty)$ such that $\delta(x)>0$ for all $x \in \overline{\partial K}$ and

$$
0<a(x) \leqslant \Theta^{n}(K, x, R) \leqslant A(x)<1
$$

whenever $x \in \overline{\partial K}$ and $0<R<\delta(x)$.

In this paper, we will frequently work with $\operatorname{Int}(K)$ and $\operatorname{Ext}(K)$. The reason is that the condition $K \in \mathcal{P}_{\mathrm{LS}}$ is not strong enough by itself to get control over topological boundaries, as demonstrated by the following simple example in the plane.

EXAMPLE 5 Let $C$ be a Jordan curve contained in an open ball $U$ in the plane. Suppose $\mathcal{H}^{2}(C)=0$, and let $K=U \backslash C$. Then $\partial_{\text {top }} K$ may have infinite length, even though $K \in \mathcal{P}_{\text {LS }}$.

However, we will be able to get a lot of control over $\partial_{\text {top }}(\operatorname{Int}(K))$ and $\partial_{\text {top }}(\operatorname{Ext}(K))($ see Theorems 10 and 11, when $K \in \mathcal{P}_{\text {LS }}$.

We will first show that, when $K \in \mathcal{P}_{\text {LS }}$, the sets $\operatorname{Int}(K)$ and $\operatorname{Ext}(K)$ are open, and each may be decomposed in a unique way into countably many pairwise disjoint, open connected components whose perimeters add up to $P(K)$. Thus, we derive the same useful decomposition formulas (5) and $(6)$ as H. Federer ([25, 4.2.25]) and L. Ambrosio, V. Caselles, S. Masnou, and J.-M. Morel ([8, Theorem 1]), except in our case the sets in the decomposition are open and connected in the usual topological sense. Since any open, connected set with finite perimeter is indecomposable ([8, Theorem 1] -also, see Section 4p, our decompositions in Theorem 6 are also decompositions into indecomposable sets, so that when working with locally simple sets we may make use of theorems and constructions from [8] as well.

Theorem 6 (On decompositions in $\mathcal{P}_{\text {LS }}$ ) Suppose $K \in \mathcal{P}_{\text {LS }}$. Then

(i) $\operatorname{Int}(K)=\left\{x: \Theta^{n}(K, x)=1\right\}$ is open.

(ii) There exists a unique, countable collection $\left\{U_{1}, U_{2}, \ldots\right\}$ of pairwise disjoint, open connected sets $U_{i} \subset \mathbb{R}^{n}$, the connected components of $\operatorname{Int}(K)$, such that $\operatorname{Int}(K)=\bigcup_{i} U_{i}$. Moreover, each $U_{i}$ has finite perimeter, and

$$
P(K)=\sum_{i} P\left(U_{i}\right)
$$

(iii) $\operatorname{Ext}(K)=\left\{x: \Theta^{n}(K, x)=0\right\}$ is open.

(iv) There exists a unique, countable collection $\left\{V_{1}, V_{2}, \ldots\right\}$ of pairwise disjoint, open connected sets $V_{i} \subset \mathbb{R}^{n}$, the connected components of $\operatorname{Ext}(K)$, such that $\operatorname{Ext}(K)=\bigcup_{i} V_{i}$. Moreover, each $V_{i}$ has finite perimeter, and

$$
P(K)=\sum_{i} P\left(V_{i}\right)
$$

(v) $\partial^{M} K$ is closed.

Proof. Let $\delta, a$, and $A$ be as in Definition 4 Suppose $x \in \operatorname{Int}(K)$. To prove (i), we will construct an $r>0$ such that any $z \in U(x, r)$ is also in $\operatorname{Int}(K)$. Since $\Theta^{n}(K, x, R)+\Theta^{n}\left(\mathbb{R}^{n} \backslash K, x, R\right)=1$, we also have

$$
0<1-A(x) \leqslant \Theta^{n}\left(\mathbb{R}^{n} \backslash K, x, R\right) \leqslant 1-a(x)<1
$$


whenever $x \in \overline{\partial K}$ and $0<R<\delta(x)$. If we had $x \in \overline{\partial K}=\overline{\partial\left(\mathbb{R}^{n} \backslash K\right)}$, then (7) would imply

$$
\limsup _{R \rightarrow 0^{+}} \Theta^{n}\left(\mathbb{R}^{n} \backslash K, x, R\right) \geqslant 1-A(x)>0,
$$

so that $\Theta^{n}\left(\mathbb{R}^{n} \backslash K, x\right) \neq 0$, contradicting $x \in \operatorname{Int}(K)$. Therefore, $x \notin \overline{\partial K}$.

We now define $D=\operatorname{dist}(x, \overline{\partial K})>0$. Since $x \in \operatorname{Int}(K)$, there exists an $r \in(0, D)$ such that $\mathcal{L}^{n}(K \cap U(x, r))>0$. If we were to have $\mathcal{L}^{n}\left(\left(\mathbb{R}^{n} \backslash K\right) \cap U(x, r)\right)>0$, then the relative isoperimetric inequality (Theorem 3 ) would give $\mathcal{H}^{n-1}(\partial K \cap U(x, r))>0$, so that $\operatorname{dist}(x, \overline{\partial K}) \leqslant \operatorname{dist}(x, \partial K)<$ $r<D$, a contradiction. Thus, $\mathcal{L}^{n}\left(\left(\mathbb{R}^{n} \backslash K\right) \cap U(x, r)\right)=0$. Now, if $z \in U(x, r)$, then we have $\mathcal{L}^{n}\left(K \cap U\left(z, r^{\prime}\right)\right)=\alpha(n)\left(r^{\prime}\right)^{n}$ whenever $r^{\prime} \in(0, r-|z-x|)$, so that $\Theta^{n}(K, z)=1$ and thus $z \in \operatorname{Int}(K)$, so (i) follows.

Since any open set in $\mathbb{R}^{n}$ can be uniquely expressed (apart from the order of the sets, of course) as a countable union of pairwise disjoint, open connected sets, the first part of (ii) holds. Now consider connected components $U_{i}$ and $U_{j}$ (with $i \neq j$ ) of $\operatorname{Int}(K)$. If $x \in \partial U_{i} \cap \partial U_{j}$, then for each positive integer $k$ we have

$$
\Theta^{n}\left(U_{k}, x\right)= \begin{cases}1 / 2 & \text { if } k=i \text { or } k=j \\ 0 & \text { otherwise }\end{cases}
$$

On the other hand, $x \in \partial U_{i} \cap \partial U_{j}$ implies $\Theta^{n}(K, x)=1$, so that $x \in \operatorname{Int}(K)$. It follows that $x \in U_{q}$ for some positive integer $q$. This implies $\Theta^{n}\left(U_{q}, x\right)=1$, which contradicts 8 . In particular, we have $\mathcal{H}^{n-1}\left(\partial U_{i} \cap \partial U_{j}\right)=0$ whenever $1 \leqslant i \neq j<\infty$, so that (since $U_{1}$ and $U_{2}$ are disjoint)

$$
P\left(U_{1} \cup U_{2}\right)=P\left(U_{1}\right)+P\left(U_{2}\right)-2 \mathcal{H}^{n-1}\left(\partial U_{1} \cap \partial U_{2}\right)=P\left(U_{1}\right)+P\left(U_{2}\right) .
$$

An easy induction argument gives $P\left(\bigcup_{i=1}^{N} U_{i}\right)=\sum_{i=1}^{N} P\left(U_{i}\right)$ for any positive integer $N$. Whenever $N \geqslant 1$, let $X_{N}=\bigcup_{i=1}^{N} U_{i}$, and $Y_{N}=\bigcup_{i=N+1}^{\infty} U_{i}=K \backslash X_{N}$. Then for each $N \geqslant 1$,

$$
\partial X_{N} \cap \partial Y_{N}=\partial\left(\bigcup_{i=1}^{N} U_{i}\right) \cap \partial\left(\bigcup_{i=N+1}^{\infty} U_{i}\right) \subset_{n-1}\left(\bigcup_{i=1}^{N} \partial U_{i}\right) \cap\left(\bigcup_{i=N+1}^{\infty} \partial U_{i}\right) \subset \bigcup_{i \neq j} \partial U_{i} \cap \partial U_{j},
$$

so that $\mathcal{H}^{n-1}\left(\partial X_{N} \cap \partial Y_{N}\right)=0$. Since $X_{N}$ and $Y_{N}$ are disjoint, we then have

$$
P(K)=P\left(X_{N}\right)+P\left(Y_{N}\right)-2 \mathcal{H}^{n-1}\left(\partial X_{N} \cap \partial Y_{N}\right)=\sum_{i=1}^{N} P\left(U_{i}\right)+P\left(Y_{N}\right),
$$

whenever $N \geqslant 1$, which implies in particular that $\sum_{i=1}^{N} P\left(U_{i}\right)$ is bounded above by $P(K)$ for any $N$. Thus, $\sum_{i=1}^{\infty} P\left(U_{i}\right) \leqslant P(K)$. Subadditivity of perimeter implies $P(K) \leqslant \sum_{i=1}^{\infty} P\left(U_{i}\right)$, so (ii) is established.

Whenever $K \in \mathcal{P}_{\text {LS }}$, we also have $\mathbb{R}^{n} \backslash K \in \mathcal{P}_{\text {LS }}$, so we may apply (i) with $K$ there replaced by $\mathbb{R}^{n} \backslash K$ to deduce that $\operatorname{Ext}(K)=\operatorname{Int}\left(\mathbb{R}^{n} \backslash K\right)$ is open, as claimed in (iii). Item (iv) follows similarly, by applying (ii) with $K$ there replaced by $\mathbb{R}^{n} \backslash K$, since $P\left(\mathbb{R}^{n} \backslash K\right)=P(K)$; and (v) follows at once from (i), (iii), and the definition of $\partial^{M} K$.

Sets in $\mathcal{P}_{\text {LS }}$ have continuous $(n-1)$-dimensional density ratio bounds as well.

THEOREM 7 (On $(n-1)$-dimensional density ratio bounds) Suppose $K \in \mathcal{P}_{\text {LS }}$, and let $\delta$ be as in Definition 4 . Then there exists a continuous function $f: \mathbb{R}^{n} \rightarrow[0, \infty)$ such that $\Theta^{n-1}(\partial K, x, R) \geqslant$ $f(x)>0$ whenever $x \in \overline{\partial K}$ and $0<R<\delta(x)$. 
Proof. The existence of $f$ follows from the bounds (4) together with the relative isoperimetric inequality (Theorem 3).

We now establish that locally simple sets of finite perimeter have boundaries which are not too wild.

THEOREM 8 (On boundary closures) Suppose $K \in \mathcal{P}_{\text {LS }}$. Then

(i) $\mathcal{H}^{n-1}(\overline{\partial K} \backslash \partial K)=0$.

(ii) $\overline{\partial K}$ has Hausdorff dimension $n-1$.

Proof. Let $\delta$ and $f$ be as in Theorem 7 Then

$$
\limsup _{R \rightarrow 0^{+}} \Theta^{n-1}(\partial K, x, R) \geqslant f(x)>0
$$

whenever $x \in \overline{\partial K}$ (and in particular whenever $x \in \overline{\partial K} \backslash \partial K$ ). It follows from [25, 2.10.19(4)] and [25, 2.10.6] that $\Theta^{n-1}(\partial K, x)=0$ for $\mathcal{H}^{n-1}$ almost all $x \in \mathbb{R}^{n} \backslash \partial K$ (and therefore for $\mathcal{H}^{n-1}$ almost all $x \in \overline{\partial K} \backslash \partial K$ in particular), so $\mathcal{H}^{n-1}(\overline{\partial K} \backslash \partial K)=0$, as claimed in (i). Since $K \in \mathcal{P}_{\text {LS }}$, we have $\mathcal{L}^{n}(K)>0$ and $\mathcal{L}^{n}\left(\mathbb{R}^{n} \backslash K\right)>0$, so the relative isoperimetric inequality (Theorem 3 ) implies $\mathcal{H}^{n-1}(\partial K)>0$. Also, $\mathcal{H}^{n-1}(\partial K)=P(K)<\infty$, so (ii) holds.

The topological boundary of a set of finite perimeter can be much larger than the reduced boundary; it can even have infinite volume and surface area, as the following example from [26] shows.

EXAMPLE $9([26,1.10])$ Let $\left\{x_{i}\right\}_{i=0}^{\infty}$ be the countably infinite set of all points in $\mathbb{R}^{n}$ having rational coordinates, and let $E=\bigcup_{i=0}^{\infty} U\left(x_{i}, 2^{-i}\right)$. Then $\mathcal{L}^{n}(E) \leqslant \alpha(n) /\left(1-2^{-n}\right)$ and $\mathcal{H}^{n-1}(\partial E)=$ $P(E) \leqslant n \alpha(n) /\left(1-2^{-(n-1)}\right)<\infty$, so $E \in \mathcal{P}$. However, since the rational points are dense in $\mathbb{R}^{n}$, we have $\bar{E}=\mathbb{R}^{n}$, and so $\partial_{\text {top }} E=\bar{E} \cap \overline{\mathbb{R}^{n} \backslash E}=\overline{\mathbb{R}^{n} \backslash E}$, so that $\mathcal{L}^{n}\left(\partial_{\text {top }} E\right)=\infty$ and of course $\mathcal{H}^{n-1}\left(\partial_{\text {top }} E\right)=\infty$.

However, when $K \in \mathcal{P}_{\text {LS }}$ we can prove a very useful result relating topological boundaries and reduced boundaries. $\operatorname{Int}(K)$ will have an $(n-1)$-dimensional topological boundary, which will coincide exactly with $\overline{\partial K}$ (Theorem 10 and exactly with the measure-theoretic boundary of $\operatorname{Int}(K)$ (Theorem 11.

TheOREM 10 (On topological boundaries) Let $K \in \mathcal{P}$. Then $\partial_{\text {top }}(\operatorname{Int}(K))=\partial_{\text {top }}(\operatorname{Ext}(K))=\overline{\partial K}$. If $K \in \mathcal{P}_{\mathrm{LS}}$, each of these has Hausdorff dimension $n-1$, contains $\partial K$, and differs from $\partial K$ (if at all) in a set having $\mathcal{H}^{n-1}$ measure 0 .

Proof. First, suppose $x \in \partial K$. Then for any $r>0$, we have $\mathcal{L}^{n}(K \cap U(x, r))>0$ and $\mathcal{L}^{n}\left(\left(\mathbb{R}^{n} \backslash K\right) \cap\right.$ $U(x, r))>0$. This first condition and Theorem 2(i) imply that $\operatorname{Int}(K) \cap U(x, r) \neq \emptyset$. The second condition and Theorem2(ii) imply that $\operatorname{Ext}(K) \cap U(x, r) \neq \emptyset$, so that $\left(\mathbb{R}^{n} \backslash \operatorname{Int}(K)\right) \cap U(x, r) \neq \emptyset$. It follows that $x \in \partial_{\text {top }}(\operatorname{Int}(K))$. Thus, $\partial K \subset \partial_{\text {top }}(\operatorname{Int}(K))$, since $x$ was arbitrary, and so $\overline{\partial K} \subset$ $\partial_{\text {top }}(\operatorname{Int}(K))$.

Now suppose $x \in \partial_{\text {top }}(\operatorname{Int}(K))$. For any $r>0, U(x, r) \cap \operatorname{Int}(K) \neq \emptyset$ and $U(x, r) \cap$ $\left(\mathbb{R}^{n} \backslash \operatorname{Int}(K)\right) \neq \emptyset$. The first of these conditions implies there exists a point $y \in U(x, r) \cap \operatorname{Int}(K)$. By Theorem 6 (i), $\operatorname{Int}(K)$ is open. Thus, $U\left(y, r^{\prime}\right) \subset U(x, r) \cap \operatorname{Int}(K)$ for some suitably small $r^{\prime}>0$, and so Theorem 2(i) implies $\mathcal{L}^{n}(K \cap U(x, r))>0$.

Next, we will show that $\mathcal{L}^{n}\left(\left(\mathbb{R}^{n} \backslash K\right) \cap U(x, r)\right)>0$. Suppose, to the contrary, that $\mathcal{L}^{n}\left(\left(\mathbb{R}^{n} \backslash K\right) \cap U(x, r)\right)=0$, so that $\mathcal{L}^{n}(K \cap U(x, r))=\alpha(n) r^{n}$. Then $\Theta^{n}(K, y)=1$ for each $y \in U(x, r)$, which implies $U(x, r) \subset \operatorname{Int}(K)$, so $U(x, r) \cap\left(\mathbb{R}^{n} \backslash \operatorname{Int}(K)\right)=\emptyset$, a contradiction. 
Since $\mathcal{L}^{n}(K \cap U(x, r))>0$ and $\mathcal{L}^{n}\left(\left(\mathbb{R}^{n} \backslash K\right) \cap U(x, r)\right)>0$, the relative isoperimetric inequality (Theorem 3 implies $\mathcal{H}^{n-1}(\partial K \cap U(x, r))>0$, so that $\operatorname{dist}(x, \partial K)<r$. Since $r$ was arbitrary, we conclude that $x \in \overline{\partial K}$, which completes the proof that $\partial_{\text {top }}(\operatorname{Int}(K))=\overline{\partial K}$. Using this result with $K$ replaced by $\mathbb{R}^{n} \backslash K$, we get

$$
\partial_{\text {top }}(\operatorname{Ext}(K))=\partial_{\text {top }}\left(\operatorname{Int}\left(\mathbb{R}^{n} \backslash K\right)\right)=\overline{\partial\left(\mathbb{R}^{n} \backslash K\right)}=\overline{\partial K} .
$$

The other stated claims follow at once from Theorem 8 .

We now show that several different notions of boundary from the literature agree for locally simple sets.

TheOREM 11 (On various notions of boundary) Suppose $K \in \mathcal{P}_{\text {LS }}$. Then

$$
\begin{aligned}
\partial^{M}(\operatorname{Int}(K)) & =\partial^{M}(\operatorname{Ext}(K))=\partial^{M} K=\overline{\partial^{M} K}=\overline{K^{1 / 2}} \\
& =\overline{\partial K}=\partial_{\text {top }}(\operatorname{Int}(K))=\partial_{\text {top }}(\operatorname{Ext}(K)) .
\end{aligned}
$$

In particular, the measure-theoretic and topological boundaries of $\operatorname{Int}(K)$ (and also of $\operatorname{Ext}(K)$ ) are precisely the same. This common boundary has Hausdorff dimension $n-1$, contains $\partial K$, and differs from $\partial K$ (if at all) in a set having $\mathcal{H}^{n-1}$ measure 0 .

Proof. $\partial^{M}(\operatorname{Int}(K))=\partial^{M}(\operatorname{Ext}(K))=\partial^{M} K$, because of Theorem 2. Because $\operatorname{Int}(K)$ and $\operatorname{Ext}(K)$ are open (Theorem 6 , $\partial^{M} K$ is closed, so the third equality holds. The sixth and seventh equalities were shown in Theorem 10 . We will now show that the measure-theoretic and topological boundaries of $\operatorname{Int}(K)$ are the same, which is equivalent (by the work above) to showing that $\overline{\partial^{M} K}=\partial_{\text {top }}(\operatorname{Int}(K))$.

Claim $1 \overline{\partial^{M} K} \subset \overline{\partial K}$.

Proof of Claim 1. Since $\overline{\partial K}$ is closed, it suffices to show that $\partial^{M} K \subset \overline{\partial K}$, which is equivalent to $\mathbb{R}^{n} \backslash \overline{\partial K} \subset \mathbb{R}^{n} \backslash \partial^{M} K=\operatorname{Int}(K) \cup \operatorname{Ext}(K)$. Suppose $x \notin \overline{\partial K}$. Since $\mathbb{R}^{n} \backslash \overline{\partial K}$ is open, $U(x, r) \subset$ $\mathbb{R}^{n} \backslash \overline{\partial K}$ for some $r>0$, and so $\mathcal{H}^{n-1}(\partial K \cap U(x, r))=0$. By the relative isoperimetric inequality (Theorem 3), this implies that either $\mathcal{L}^{n}(K \cap U(x, r))=0$ or $\mathcal{L}^{n}\left(\left(\mathbb{R}^{n} \backslash K\right) \cap U(x, r)\right)=0$; otherwise it would follow that $\mathcal{H}^{n-1}(\partial K \cap U(x, r))>0$. In case $\mathcal{L}^{n}(K \cap U(x, r))=0$, we have $\mathcal{L}^{n}\left(\left(\mathbb{R}^{n} \backslash K\right) \cap U(x, r)\right)=\alpha(n) r^{n}$, so at each point of $U(x, r)$ the $n$-density of $\mathbb{R}^{n} \backslash K$ is 1 , so $x \in U(x, r) \subset \operatorname{Ext}(K) \subset \mathbb{R}^{n} \backslash \partial^{M} K$. Similarly, in case $\mathcal{L}^{n}\left(\left(\mathbb{R}^{n} \backslash K\right) \cap U(x, r)\right)=0$, we deduce $x \in \operatorname{Int}(K) \subset \mathbb{R}^{n} \backslash \partial^{M} K$.

Claim $2 \overline{\partial^{M} K}=\partial_{\text {top }}(\operatorname{Int}(K))$.

Proof of Claim 2. Suppose $x \in \overline{\partial^{M} K}$. Since $K \in \mathcal{P}_{\mathrm{LS}}$, there exist continuous functions $\delta, a, A$ : $\mathbb{R}^{n} \rightarrow[0, \infty)$ such that $\delta(x)>0$ for all $x \in \overline{\partial K}$ and

$$
0<a(x) \leqslant \Theta^{n}(K, x, r) \leqslant A(x)<1
$$

whenever $x \in \overline{\partial K}$ and $0<r<\delta(x)$. By Claim $1, x \in \overline{\partial K}$, so 9 implies that $\mathcal{L}^{n}(K \cap U(x, r))>0$ and $\mathcal{L}^{n}\left(\left(\mathbb{R}^{n} \backslash K\right) \cap U(x, r)\right)>0$ for all $r>0$. By Theorem 2 for any $r>0$ we have $\mathcal{L}^{n}(\operatorname{Int}(K) \cap$ $U(x, r))>0$ and $\mathcal{L}^{n}(\operatorname{Ext}(K) \cap U(x, r))>0$, and so it follows that $U(x, r)$ contains points in $\operatorname{Int}(K)$ and in $\mathbb{R}^{n} \backslash \operatorname{Int}(K)$ for each $r>0$, so that $x \in \partial_{\text {top }}(\operatorname{Int}(K))$.

To complete the proof of the claim, we will show that $\partial_{\text {top }}(\operatorname{Int}(K)) \subset \overline{\partial^{M} K}$. Using the fact that $\partial^{M} K$ is closed, and taking complements, this is equivalent to showing that $\operatorname{Int}(K) \cup \operatorname{Ext}(K) \subset$ 
$\mathbb{R}^{n} \backslash \partial_{\text {top }}(\operatorname{Int}(K))$. Suppose $x \in \operatorname{Int}(K)$. Since $\operatorname{Int}(K)$ is open, there exists an $r>0$ such that $U(x, r) \subset \operatorname{Int}(K)$. It follows that $x \notin \partial_{\text {top }}(\operatorname{Int}(K))$, since otherwise each $U(x, r)$ would contain points inside and outside $\operatorname{Int}(K)$. Since $\operatorname{Ext}(K)$ is also open, $x \in \operatorname{Ext}(K)$ implies $U(x, r) \subset \operatorname{Ext}(K)$ for some $r>0$, which implies $x \notin \partial_{\text {top }}(\operatorname{Int}(K))$ as before.

It remains to show that $\overline{K^{1 / 2}}$ is also equal to the other boundary terms. This follows from the fact that $\overline{\partial K}=\overline{\partial^{M} K}$, because of Theorem 2(v).

REMARK 12 (Other boundaries) Of course, $\partial K$ and $K^{1 / 2}$ need not be closed, even if $K \in \mathcal{P}_{\text {LS }}$ simply take $K$ to be a cube. However, if $\partial K$ is closed for some $K \in \mathcal{P}_{\text {LS }}$ then $\partial K$ and $K^{1 / 2}$ are each equal to the various boundary terms in Theorem 11 as are $\partial(\operatorname{Int}(K))$ and $\partial(\operatorname{Ext}(K))$ because of Theorem 2

If we use $K$ instead of $\operatorname{Int}(K)$, the topological boundary may not equal the measure-theoretic boundary, even if $K \in \mathcal{P}_{\mathrm{LS}}$, as the following example shows.

EXAMPLE 13 Take $K$ to be a closed ball minus its center; the center is part of $\partial_{\text {top }} K$ but is not part of $\partial^{M} K$ (since in fact it is in $\operatorname{Int}(K)$ ), so $\partial_{\text {top }} K \neq \partial^{M} K$ even though $\partial_{\text {top }} K$ is closed and $K \in \mathcal{P}_{\text {LS }}$.

The next example suggests the importance of local simplicity if we want topological and measure-theoretic boundaries to be the same. In the example, $\partial_{\text {top }}(\operatorname{Int}(K)) \neq \partial^{M}(\operatorname{Int}(K))$ even though the $n$-dimensional density of $K$ fails to stay away from 0 and 1 only at a single point, the origin.

EXAMPLE 14 Let $Q_{1}$ be the open cube in $\mathbb{R}^{n}$ with edge length 2 , with faces perpendicular to the axes, and centered at $(-1,0, \ldots, 0)$. Let $Q_{2}$ be the open cube in $\mathbb{R}^{n}$ with edge length 2 , with faces perpendicular to the axes, and centered at $(1,0, \ldots, 0)$. Let $U_{1}$ and $U_{2}$ be the open unit balls inscribed in $Q_{1}$ and $Q_{2}$, respectively, and let $K=U_{1} \cup U_{2}$. Then $\operatorname{Int}(K)=U_{1} \cup U_{2} \cup\{\mathbf{0}\}$, where $\mathbf{0}$ is the origin. We note that $\mathbf{0} \in \partial_{\text {top }}(\operatorname{Int}(K))$ but $\mathbf{0} \notin \partial^{M}(\operatorname{Int}(K))$, since $\mathbf{0} \in \operatorname{Int}(K)=\operatorname{Int}(\operatorname{Int}(K))$.

Theorem 11 allows us to strengthen the conclusion of Theorem 2(vii), if the set is locally simple. The following result justifies intuitive arguments in which we think of a set in $\mathbb{R}^{n}$ as having an open interior, an open exterior, and a closed, $(n-1)$-dimensional boundary.

Proposition 15 (On partitions by a set in $\mathcal{P}_{\text {LS }}$ ) Each $K \in \mathcal{P}_{\text {LS }}$ partitions $\mathbb{R}^{n}$ exactly into an open interior, $\operatorname{Int}(K)$, an open exterior, $\operatorname{Ext}(K)$, and a closed $(n-1)$-dimensional boundary, $\overline{\partial K}$.

Proof. The result follows at once from the definition of $\partial^{M} K$, and from the fact that $\overline{\partial K}=\partial^{M} K$ when $K \in \mathcal{P}_{\text {LS }}$.

\section{Connectedness and indecomposability}

In this section we will compare the topological notion of connectedness with the measure-theoretic notion of indecomposability, introduced by H. Federer ([25, 4.2.25]) in the context of integral currents, and specialized to sets of finite perimeter by L. Ambrosio, V. Caselles, S. Masnou, and J.-M. Morel in [8].

DeFinition $16([\overline{8}])$ A set $K \in \mathcal{P}$ is indecomposable provided $K$ cannot be written as $K=$ $A \cup B$, where $A \cap B=\emptyset, \mathcal{L}^{n}(A)>0, \mathcal{L}^{n}(B)>0$, and $P(K)=P(A)+P(B)$. 
The integral current $[K]$ (see (2p) is indecomposable in the sense of [25, 4.2.25] if and only if the set $K$ is indecomposable. Neither indecomposability nor connectedness implies the other in general, as the following theorem shows.

THEOREM 17 (On connectedness and indecomposability) Regardless of whether a set $K \in \mathcal{P}$ is connected or not, it may be indecomposable or decomposable. This remains true even if we further require $K \in \mathcal{P}_{\text {LS }}$.

Proof. Let $Q_{1}, Q_{2}, U_{1}$, and $U_{2}$ be as in Example 14 Each of the four sets below is in $\mathcal{P}_{\text {LS }}$.

\begin{tabular}{lll}
\hline & Indecomposable & Decomposable \\
\hline Connected & $U(\mathbf{0}, 1)$ & $U_{1} \cup U_{2} \cup\{\mathbf{0}\}$ \\
\hline Not connected & $Q_{1} \cup Q_{2}$ & $U(\mathbf{0}, 1) \cup U((5,0, \ldots, 0), 1)$ \\
\hline
\end{tabular}

L. Ambrosio, V. Caselles, S. Masnou, and J.-M. Morel showed that, for open subsets of $\mathbb{R}^{n}$ having finite perimeter, connectedness does imply indecomposability ([8, Proposition 2]), but not conversely, as their example of an open disk minus its diameter demonstrates.

Indecomposable sets need not be equal (even mod $\mathcal{L}^{n}$ negligible sets) to open, connected sets (see [8, Example 2]). The following very general proposition shows that the situation is quite different for disconnected sets.

Proposition 18 (Equivalence to disconnected sets) Suppose $K$ is any Lebesgue measurable subset of $\mathbb{R}^{n}$. Then there exists a disconnected set $K^{*} \subset \mathbb{R}^{n}$ such that $K={ }_{n} K^{*}$.

Proof. If $\mathcal{L}^{n}(K)=0$, we can take $K^{*}$ to be any set consisting of two distinct points in $\mathbb{R}^{n}$. If $\mathcal{L}^{n}(K)>0$, then there exist $p, q \in K$ with $p \neq q$. Let $H$ be the hyperplane orthogonal to and bisecting $\overline{p q}$. Then $K^{*}=K \backslash H$ is disconnected and satisfies $K={ }_{n} K^{*}$.

Next, we will compare our decomposition theorem, Theorem 6 , with the theorems of H. Federer ([25, 4.2.25]) and L. Ambrosio, V. Caselles, S. Masnou, and J.-M. Morel ([8, Theorem 1]). Since any open, connected set with finite perimeter is indecomposable ([8, Theorem 1]), our decompositions in Theorem 6 are also decompositions into indecomposable sets. For comparison purposes, we will essentially quote [8, Theorem 1], changing the notation so as to agree with our own.

Theorem 19 (On decompositions in $\mathcal{P}$; [25, 4.2.25], [8, Theorem 1]) Suppose $E \in \mathcal{P}$. Then there exists a unique countable family, $C C^{M}(E)$, of pairwise disjoint indecomposable sets $\left\{E_{i}\right\}_{i \in I}$ such that $\mathcal{L}^{n}\left(E_{i}\right)>0$ for each $i$, and $P(E)=\sum_{i} P\left(E_{i}\right)$. Moreover, $\mathcal{H}^{n-1}\left(\operatorname{Int}(E) \backslash \bigcup_{i} \operatorname{Int}\left(E_{i}\right)\right)$ $=0$, and the $E_{i}$ 's are maximal indecomposable sets, i.e., any indecomposable set $F \subset E$ is $\mathcal{H}^{n}$ almost contained in some set $E_{i}$.

We now show that, for locally simple sets, their decomposition theorems and ours give the same decomposition, i.e., the decompositions into sets of finite perimeter will also be decompositions into open, connected sets.

THEOREM 20 (On the equivalence of decompositions) Suppose $K \in \mathcal{P}_{\text {LS }}$. Let $\left\{U_{1}, U_{2}, \ldots\right\}$ and $\left\{V_{1}, V_{2}, \ldots\right\}$ be the (unique) countable, pairwise disjoint collections of open, connected sets defined in Theorem 6 (ii) and (iv) respectively. Then $C C^{M}(\operatorname{Int}(K))=\left\{U_{1}, U_{2}, \ldots\right\}$, and $C C^{M}(\operatorname{Ext}(K))=$ $\left\{V_{1}, V_{2}, \ldots\right\}$.

Proof. According to Theorem 2 of [8], the collection of connected components and the collection of maximal indecomposable sets of an open set $A \subset \mathbb{R}^{n}$ are the same provided $\mathcal{H}^{n-1}\left(\partial_{\text {top }} A\right)=$ 
$\mathcal{H}^{n-1}\left(\partial^{M} A\right)$. It suffices to show that this condition is satisfied when $A=\operatorname{Int}(K)$ and when $A=$ $\operatorname{Ext}(K)$ for $K \in \mathcal{P}_{\text {LS }}$. We calculate

$$
\begin{aligned}
\mathcal{H}^{n-1}\left(\partial_{\text {top }}(\operatorname{Int}(K))\right) & =\mathcal{H}^{n-1}(\overline{\partial K}) & & (\text { Theorem } 10) \\
& =\mathcal{H}^{n-1}(\partial K) & & (\text { Theorem } 8(\mathrm{i})) \\
& =\mathcal{H}^{n-1}(\partial(\operatorname{Int}(K))) & & (\text { Theorem } 2 \text { iii })) \\
& =\mathcal{H}^{n-1}\left(\partial^{M}(\operatorname{Int}(K))\right) & & (\text { Theorem 2 vi })) .
\end{aligned}
$$

Replacing $K$ by $\mathbb{R}^{n} \backslash K$ above gives $\mathcal{H}^{n-1}\left(\partial_{\text {top }}(\operatorname{Ext}(K))\right)=\mathcal{H}^{n-1}\left(\partial^{M}(\operatorname{Ext}(K))\right)$.

\section{Locally simple sets and $L^{1}$ TV-minimization}

Variational methods have become increasingly popular in image processing. The ROF model of L. Rudin, S. Osher, and E. Fatemi [35] has been particularly successful, and it has led to a considerable amount of related research in the last two decades.

In the ROF model, we have an open connected set $\Omega \subset \mathbb{R}^{n}(n \geqslant 2)$ with Lipschitz boundary, and a possibly noisy image $s \in L^{2}(\Omega)$. The goal is to obtain a reconstructed image $u \in L^{2}(\Omega)$ which is "close" to $s$ in some sense, and which is less noisy than $s$. Let

$$
E_{\lambda}^{*}(u)=\operatorname{TV}(u, \Omega)+\lambda \int_{\Omega}(u-s)^{2} \mathrm{~d} \mathcal{L}^{n}
$$

whenever $\lambda>0$. The energy $E_{\lambda}^{*}(u)$ is lower semicontinuous and strictly convex, and $E_{\lambda}^{*}(u)$ has a unique minimizer for each $\lambda$.

The integral in the second summand in (10) is called the fidelity term, as it is a measure of deviation of $u$ from $s$. Large values of $\lambda$ result in reconstructed images $u$ which are quite close to $s$ but which may not be too regular. Small values of $\lambda$ result in images $u$ that more closely resemble TV-minimizers, so they are more regular but may be rather different from $s$. Thus, $\lambda$ determines the trade-off between fidelity and regularity; the method is therefore quite flexible and adaptable.

The ROF model has drawbacks, however, such as loss of contrast even for noise-free, texturefree images in the plane. For example, if $s=\chi_{D}$, where $D=\left\{x \in \mathbb{R}^{2}:|x| \leqslant r\right\}$ for some $r>0$, then $u=0$ if $\lambda \leqslant 1 / r$ while $u=\left(1-(\lambda r)^{-1}\right) \chi_{D}$ if $\lambda>1 / r$ (cf. [20]). We cannot recover the original image $s$ no matter which value of $\lambda$ we use.

Subsequently, many variants and extensions of the model have been considered, in both discrete and continuous settings, theoretically and computationally. One such model is $L^{1} \mathrm{TV}$-minimization, considered by S. Alliney and M. Nikolova (see, for example, [4] and [34]) in a discrete setting, and later considered by T. Chan and S. Esedoglu [20] and subsequently by W. Allard (see, for example, [1]) in a continuous setting for $n \geqslant 2$. We will restrict our attention hereafter to the continuous case, with $n \geqslant 2$, and we will primarily adopt the notation of W. Allard from [1].

In $L^{1} \mathrm{TV}$-minimization, the $L^{2}$ norm fidelity term in $E_{\lambda}^{*}(u)$ above is replaced by an eponymous $L^{1}$ norm fidelity term, so that the energy to be minimized is

$$
E_{\lambda}(u)=\mathrm{TV}(u, \Omega)+\lambda \int_{\Omega}|u-s| \mathrm{d} \mathcal{L}^{n},
$$

where $\lambda>0$. 
The change from an $L^{2}$ norm to an $L^{1}$ norm results in major qualitative differences between the two procedures. For instance, consider the disk example above, in which $s=\chi_{D}$. Using $L^{1}$ TV-minimization, we get unique solutions unless $\lambda=2 / r$; specifically, $u=0$ if $\lambda<2 / r$, and $u=s$ if $\lambda>2 / r$. However, if $\lambda=2 / r$ then $u=c s$ is a solution for each $c \in[0,1]$ (see [20]).

This simple example highlights advantages and disadvantages of $L^{1}$ TV-minimization, as compared to the ROF model. There are advantages with regard to maintaining contrast but disadvantages concerning uniqueness and stability of solutions: as this example demonstrates, slight perturbations of $\lambda$ may result in sudden and major changes in $u$, for critical values of $\lambda$. The lack of uniqueness is due to the lack of strict convexity of $E_{\lambda}(u)$. Depending on the application, however, lack of uniqueness may be considered a feature of the model rather than a drawback.

For an open subset $\Omega$ of $\mathbb{R}^{n}$, we let

$$
I(\Omega)=\left\{f: f \in L^{1}(\Omega) \cap L^{\infty}(\Omega), f(x) \geqslant 0 \text { for all } x \in \Omega\right\}
$$

denote our collection of admissible observed images $s$, as in [1].

Whenever $f \in L_{\text {loc }}^{1}(\Omega)$ and $K \subset \Omega$ is compact, we define (cf. [1, 1.1])

$$
\begin{aligned}
\mathbf{k}(f, K) & =\left\{g: g \in L_{\mathrm{loc}}^{1}(\Omega), \text { and }\{g \neq f\} \subset_{n} K\right\}, \\
\mathbf{k}(f) & =\bigcup\{\mathbf{k}(f, K): K \subset \Omega \text { and } K \text { is compact }\} .
\end{aligned}
$$

Whenever $s \in I(\Omega)$, we let

$$
\mathbf{m}_{\lambda}^{\mathrm{loc}}(s, \Omega)=\left\{u: u \in I(\Omega), \operatorname{TV}(u, \Omega)<\infty, \text { and } E_{\lambda}(u) \leqslant E_{\lambda}(f) \forall f \in I(\Omega) \cap \mathbf{k}(u)\right\}
$$

denote the collection of local $L^{1} \mathrm{TV}$-minimizers for $s$ in $\Omega$. We will investigate the local simplicity of the super-level sets $E_{y}=\{u>y\}$, where $u$ is an $L^{1}$ TV-minimizer for an initial (possibly noisy) image $s \in I(\Omega)$.

For $0 \leqslant \lambda<\infty$ we define $\mathcal{B}_{\lambda}(\Omega)$ to be the set of all $f$ in $\operatorname{BV}_{\text {loc }}(\Omega)$ such that, for each compact $K \subset \Omega$ (cf. [1, 1.5]),

$$
\operatorname{TV}(f, K) \leqslant \operatorname{TV}(g, K)+\lambda \int_{\Omega}|f-g| \mathrm{d} \mathcal{L}^{n} \quad \text { whenever } g \in \mathbf{k}(f, K) .
$$

We similarly define $\mathcal{C}_{\lambda}(\Omega)$ to be the set of all $\mathcal{L}^{n}$ measurable subsets $A$ of $\Omega$ such that $\chi_{A} \in$ $\mathrm{BV}_{\text {loc }}(\Omega)$ and, for each compact $K \subset \Omega$ (cf. [1, 1.5]),

$$
\operatorname{TV}\left(\chi_{A}, K\right) \leqslant \operatorname{TV}\left(\chi_{B}, K\right)+\lambda \int_{\Omega}\left|\chi_{A}-\chi_{B}\right| \mathrm{d} \mathcal{L}^{n} \text { whenever } B \subset \Omega \text { with } \chi_{B} \in \mathbf{k}\left(\chi_{A}, K\right)
$$

Whenever $A$ is an $\mathcal{L}^{n}$ measurable subset of $\Omega$, we have ([1, Theorem 5.3(iv)])

$$
A \in \mathcal{C}_{\lambda}(\Omega) \Leftrightarrow \chi_{A} \in \mathcal{B}_{\lambda}(\Omega)
$$

We now show that super-level sets and sub-level sets of $L^{1} \mathrm{TV}$-minimizers satisfy continuous lower density ratio bounds. 
LEMMA 21 (Lower density ratio bounds for $L^{1} \mathrm{TV}$-minimizers)

Assumptions:

(a) $n \geqslant 2$.

(b) $\Omega$ is a non-empty open subset of $\mathbb{R}^{n}$.

(c) $s \in I(\Omega)$ is an initial (possibly noisy) image.

(d) $\lambda \in(0, \infty)$.

(e) $u \in \mathbf{m}_{\lambda}^{\text {loc }}(s, \Omega)$ is an $L^{1} \mathrm{TV}$-minimizer for $s$ in $\Omega$.

(f) For each $y \in \mathbb{R}$, let $E_{y}=\{u>y\}$, and let $\left[E_{y}\right]$ be the multiplicity one integral $n$-current associated with $E_{y}$, as defined by (2).

Conclusions: For any $y \in \mathbb{R}$,

(i) If $a \in \operatorname{spt}\left[E_{y}\right]$, then for all $r \in\left(0, \operatorname{dist}\left(a, \mathbb{R}^{n} \backslash \Omega\right)\right)$, we have

$$
(1+\lambda r) \mathcal{L}^{n}\left(E_{y} \cap U(a, r)\right) \geqslant e^{-\lambda r} \cdot \frac{\alpha(n-1)}{n} r^{n} .
$$

(ii) If $a \in \operatorname{spt}\left[\Omega \backslash E_{y}\right]$, then for all $r \in\left(0, \operatorname{dist}\left(a, \mathbb{R}^{n} \backslash \Omega\right)\right)$, we have

$$
(1+\lambda r) \mathcal{L}^{n}\left(\left(\Omega \backslash E_{y}\right) \cap U(a, r)\right) \geqslant e^{-\lambda r} \cdot \frac{\alpha(n-1)}{n} r^{n} .
$$

Proof. If $a \in \mathbb{R}^{n} \backslash \Omega$ then both conclusions hold vacuously, so we hereafter suppose $a \in \Omega$. For the case of $L^{1}$ TV-minimization, we apply Proposition 1.1 of [1] with $\epsilon=1 / \lambda$ there to deduce that $u \in \mathcal{B}_{\lambda}(\Omega)$. Therefore, $E_{y}=\{u>y\} \in \mathcal{C}_{\lambda}(\Omega)$ by [1, Theorem 5.3(i)]. In particular, the hypotheses of Theorem 5.4 of [1] are satisfied with $D$ there replaced by $E_{y}$. The bound $(15)$ is the one given in Theorem 5.4(v) of [1], whenever $a \in \Omega \cap \operatorname{spt}\left[E_{y}\right]$ and $0<r<\operatorname{dist}\left(a, \mathbb{R}^{n} \backslash \Omega\right)$.

Since $E_{y} \in \mathcal{C}_{\lambda}(\Omega),\left[14\right.$ implies $\chi_{E_{y}} \in \mathcal{B}_{\lambda}(\Omega)$. We next apply Theorem 5.1 of [1] to deduce that $\chi_{\Omega \backslash E_{y}}=1-\chi_{E_{y}} \in \mathcal{B}_{\lambda}(\Omega)$, so that $\Omega \backslash E_{y} \in \mathcal{C}_{\lambda}(\Omega)$. This enables us to use Theorem 5.4(v) of [1] with $D$ there replaced by $\Omega \backslash E_{y}$ to deduce (ii).

The next lemma ensures that the upper and lower density ratio bounds (19) and (20) will be strictly between 0 and 1 when $x \in \overline{\partial E_{y}}$, as required for local simplicity of $E_{y}$.

LEMMA 22 For any integer $n \geqslant 2$, and for any non-negative real numbers $\lambda$ and $r$, we define

$$
b(\lambda, r)=\frac{\alpha(n-1)}{n \alpha(n)} \cdot \frac{e^{-\lambda r}}{1+\lambda r} .
$$

If $\lambda>0$ then $b(\lambda, r)$ is strictly decreasing as a function of $r$, while if $r>0$ then $b(\lambda, r)$ is strictly decreasing as a function of $\lambda$; moreover, for all $\lambda, r \geqslant 0$ we have

$$
0<b(\lambda, r) \leqslant \frac{\alpha(n-1)}{n \alpha(n)}<\frac{1}{2} .
$$

Proof. The first claims hold since the function $f(x)=e^{-x} /(1+x)$ satisfies $f^{\prime}(x)<0$ for all $x \geqslant 0$, and the first two inequalities of 18 hold since $0<f(x) \leqslant 1$ when $x \geqslant 0$. Because the area integrand is strictly convex, if we replace a unit ball in $\mathbb{R}^{n}$ by a half-ball (by intersecting the ball with a half-space passing through its center), the surface area must decrease. Thus, the surface area change $\alpha(n-1)-(1 / 2) n \alpha(n)$ must be negative, and this implies the rightmost inequality of (18). 
We can now establish the local simplicity of the sets $E_{y}=\{u>y\}$, for $L^{1}$ TV-minimizers $u$. We will treat the cases $\Omega=\mathbb{R}^{n}$ and $\Omega \neq \mathbb{R}^{n}$ separately since our proofs and our upper and lower density ratio bounds will differ.

THEOREM 23 (Super-level sets of $L^{1}$ TV-minimizers in $\mathbb{R}^{n}$ are locally simple) Suppose that assumptions (a)-(f) of Lemma 21 hold with $\Omega=\mathbb{R}^{n}$. Then for each $y \in \mathbb{R}$ either $\partial E_{y}=\emptyset$ or $E_{y}$ is locally simple. In the latter case, $E_{y}$ has each of the regularity and structural properties given in Sections 3 and 4 and for each $\theta>0$ the set $E_{y}$ also satisfies

$$
0<b(\lambda, \theta) \leqslant \Theta^{n}\left(E_{y}, x, r\right) \leqslant 1-b(\lambda, \theta)<1
$$

whenever $x \in \overline{\partial E_{y}}$ and $0<r<\theta$. Here, $b$ is the function from 17 .

Proof. Suppose $\theta>0$ and $\partial E_{y} \neq \emptyset$. We will show that $E_{y}$ satisfies Definition 4 with $\delta(x) \equiv \theta$, $a(x) \equiv b(\lambda, \theta)$, and $A(x) \equiv 1-b(\lambda, \theta)$ for all $x \in \mathbb{R}^{n}$. [18) guarantees that $0<a(x)<A(x)<1$ whenever $x \in \overline{\partial E_{y}}$ and $0<r<\delta(x)$.

In the proof of Lemma 21 we showed that for all $y \in \mathbb{R}$ the set $E_{y}$ is in $\mathcal{C}_{\lambda}(\Omega)$, so that in particular $E_{y}$ is a Lebesgue measurable subset of $\Omega$ having locally finite perimeter. This allows us to apply Proposition 1 , together with (15) and Lemma 22, to deduce that

$$
\Theta^{n}\left(E_{y}, x, r\right)=\frac{\mathcal{L}^{n}\left(E_{y} \cap U(x, r)\right)}{\alpha(n) r^{n}} \geqslant b(\lambda, r)>b(\lambda, \theta)>0
$$

whenever $x \in \overline{\partial E_{y}}$ and $0<r<\theta$. Similarly, Proposition 1, 16 , and Lemma 22 imply that

$$
\Theta^{n}\left(\mathbb{R}^{n} \backslash E_{y}, x, r\right)=\frac{\mathcal{L}^{n}\left(\left(\mathbb{R}^{n} \backslash E_{y}\right) \cap U(x, r)\right)}{\alpha(n) r^{n}} \geqslant b(\lambda, r)>b(\lambda, \theta)>0
$$

whenever $x \in \overline{\partial\left(\mathbb{R}^{n} \backslash E_{y}\right)}=\overline{\partial E_{y}}$ and $0<r<\theta$. For such $x$ and $r$ we thus have

$$
\Theta^{n}\left(E_{y}, x, r\right)=1-\Theta^{n}\left(\mathbb{R}^{n} \backslash E_{y}, x, r\right)<1-b(\lambda, \theta)<1 .
$$

EXAMPLE 24 Consider one of the most common cases in applications: $n=2$. We have $b(\lambda, \theta)=$ $(1 / \pi) f(\lambda \theta)$, where $f(x)=e^{-x} /(1+x)$. For any $\lambda>0$ we can have the density ratios $\Theta^{n}\left(E_{y}, x, r\right)$ uniformly bounded (approximately) between $1 / \pi$ and $1-1 / \pi$ if we take $\theta$ to be sufficiently small. Provided our resolution is large enough, taking $\theta$ to be small is not a problem.

When $\lambda>0$ and $\theta>0$ are fixed, Theorem 23 gives constant lower and upper bounds to the $n$ dimensional density ratios $\Theta^{n}\left(E_{y}, x, r\right)$ at points $x \in \overline{\partial E_{y}}$, out to radius $\theta$. Since Theorem 23 holds for any size scale $\theta$, we can use it for several different values of $\theta$, chosen for instance according to the size of the noise or of various features of interest.

We now consider arbitrary non-empty open subsets $\Omega$ of $\mathbb{R}^{n}$, with $\Omega \neq \mathbb{R}^{n}$. We need to keep $\partial E_{y}$ away from $\partial \Omega$, which may in general be highly irregular.

THEOREM 25 (Super-level sets of $L^{1}$ TV-minimizers in $\Omega$ are locally simple) Suppose that assumptions (a)-(f) of Lemma 21 hold, and also $\Omega \neq \mathbb{R}^{n}$. Then, for each $y \in \mathbb{R}$ for which $\operatorname{spt}\left[E_{y}\right] \subset \Omega$, either $\partial E_{y}=\varnothing$ or $E_{y}$ is locally simple. In the latter case, $E_{y}$ has each of the regularity and structural properties given in Sections 3 and 4 , and $E_{y}$ also satisfies

$$
0<b(\lambda, \delta(x)) \leqslant \Theta^{n}\left(E_{y}, x, r\right) \leqslant 1-b(\lambda, \delta(x))<1
$$

whenever $x \in \overline{\partial E_{y}}$ and $0<r<\delta(x)$. Here, $b$ is the function from $\sqrt[17]{17}$, and $\delta(x)=\operatorname{dist}\left(x, \mathbb{R}^{n} \backslash \Omega\right)$ for all $x \in \mathbb{R}^{n}$. 
Proof. Suppose $y$ satisfies $\operatorname{spt}\left[E_{y}\right] \subset \Omega$ and that $\partial E_{y} \neq \emptyset$. We will show that $E_{y}$ satisfies Definition 4 with $\delta, a$, and $A$ defined for all $x \in \mathbb{R}^{n}$ by $\delta(x)=\operatorname{dist}\left(x, \mathbb{R}^{n} \backslash \Omega\right), a(x)=b(\lambda, \delta(x))$, and $A(x)=1-b(\lambda, \delta(x))$.

By Proposition $1, \overline{\partial E_{y}} \subset \operatorname{spt}\left[E_{y}\right] \subset \Omega$, so that $\overline{\partial E_{y}}$ and $\mathbb{R}^{n} \backslash \Omega$ are disjoint closed sets. It follows that $\delta(x)>0$ for each $x \in \overline{\partial E_{y}}$, as required. $\delta$ is Lipschitz with $\operatorname{Lip} \delta=1$, so in particular it is continuous on $\mathbb{R}^{n}$, and therefore so are $a$ and $A$.

As in the proof of Theorem 23, $E_{y} \in \mathcal{C}_{\lambda}(\Omega)$ for each $y \in \mathbb{R}$. It follows from Proposition 1. [15, and Lemma 22 that

$$
\Theta^{n}\left(E_{y}, x, r\right)=\frac{\mathcal{L}^{n}\left(E_{y} \cap U(x, r)\right)}{\alpha(n) r^{n}} \geqslant b(\lambda, r)>b(\lambda, \delta(x))>0
$$

whenever $x \in \overline{\partial E_{y}}$ and $0<r<\delta(x)$. Similarly, Proposition 1, 16, and Lemma 22 imply that

$$
\Theta^{n}\left(\mathbb{R}^{n} \backslash E_{y}, x, r\right)=\frac{\mathcal{L}^{n}\left(\left(\mathbb{R}^{n} \backslash E_{y}\right) \cap U(x, r)\right)}{\alpha(n) r^{n}} \geqslant b(\lambda, r)>b(\lambda, \delta(x))>0
$$

whenever $x \in \overline{\partial\left(\mathbb{R}^{n} \backslash E_{y}\right)}=\overline{\partial E_{y}}$ and $0<r<\delta(x)$. Finally, for such $x$ and $r$ we have

$$
\Theta^{n}\left(E_{y}, x, r\right)=1-\Theta^{n}\left(\mathbb{R}^{n} \backslash E_{y}, x, r\right)<1-b(\lambda, \delta(x))<1 .
$$

REMARK 26 When $\Omega$ is bounded, as is often the case in applications, $\overline{\partial E_{y}}$ is compact. So, when $\operatorname{spt}\left[E_{y}\right] \subset \Omega$, there exists a constant $c(y)>0$ such that $\delta(x) \geqslant c(y)$ for all $x \in \overline{\partial E_{y}}$. In particular, the density ratio bounds from Theorem 25 hold at least out to the uniform radius $c(y)$ at each $x \in \overline{\partial E_{y}}$.

Because $b(\lambda, r)$ is decreasing as a function of $\lambda$, for fixed $r$, in both Theorem 23 and Theorem 25 we get tighter density ratio bounds if we decrease $\lambda$. This makes practical and intuitive sense since, when $\lambda$ decreases, the function $u$ tries harder to minimize total variation, so we expect more regularity.

For other regularity results, concerning smoothness and curvature bounds, see [1]-[3].

\section{Conclusion}

Local simplicity is a quite general condition, satisfied by many variational problems in the finite perimeter or BV setting, in fields such as materials science, biology, image processing, and oncology. It implies some weak regularity results, as given in Sections 3 and 4 Locally simple sets have many properties - measure-theoretic, topological, and structural — which are desirable for both theory and applications. For example, when $K \in \mathcal{P}_{\mathrm{LS}}$ the topological boundary of $\operatorname{Int}(K)$, the measure-theoretic boundary of $\operatorname{Int}(K)$, and the closure of the reduced boundary of $\operatorname{Int}(K)$ agree exactly, and each is an $(n-1)$-dimensional closed set; this common boundary partitions the rest of $\mathbb{R}^{n}$ into an open interior, $\operatorname{Int}(K)$, and an open exterior, $\operatorname{Ext}(K)$. When $K \in \mathcal{P}_{\mathrm{LS}}$, the usual decomposition of $\operatorname{Int}(K)$ into connected components and the decomposition of $\operatorname{Int}(K)$ into maximal indecomposable sets coincide, so that standard results about connectedness from topology and results about indecomposability from [25] and from [8] may all be used.

The explicit density ratio bounds one establishes to verify that local simplicity holds are of practical value as well. Since they provide concrete criteria which minimizers must satisfy if the 
computational scheme is correct, they can be used, for example, to check the accuracy of computer implementations of variational procedures, or even (as in the case of $L^{1} \mathrm{TV}$-minimization) to eliminate some noise or uncertainty in the minimizers.

\section{Acknowledgements}

I would like to express my gratitude to the reviewers, whose valuable suggestions helped to significantly improve this paper.

\section{REFERENCES}

1. Allard, W. K. Total variation regularization for image denoising, I. Geometric theory. SIAM J. Math. Anal. 39 (2007), 1150-1190. Zbl 1185.49047 MR 2368898

2. Allard, W. K. Total variation regularization for image denoising, II. Examples. SIAM J. Imaging Sci. 1 (2008), 400-417. Zbl 1185.49048 MR 2486028

3. Allard, W. K. Total variation regularization for image denoising, III. Examples. SIAM J. Imaging Sci. 2 (2009), 532-568. Zbl 1175.49038 MR 2519922

4. Alliney, S. Digital filters as absolute norm regularizers. IEEE Trans. Signal Processing 40 (1992), 1548-1562. Zbl 0859.93037

5. Almgren, F. J., Jr. Existence and regularity almost everywhere of solutions to elliptic variational problems with constraints. Mem. Amer. Math. Soc. 165 (1976). Zbl 0327.49043 MR 0420406

6. Almgren, F. J., Jr., \& TAYlor, J. E. Soap bubble clusters. Forma 11 (1996), 199-207; reprinted in: The Kelvin Problem, Denis Weaire (ed.), Taylor and Francis, London (1996), 37-45. Zbl 0900.49033 MR 1487207

7. Almgren, F. J., Jr., TAYlor, J. E., \& WANG, L. Curvature driven flows: a variational approach. SIAM J. Control Optim. 31 (1993), 387-438. Zbl 0783.35002 MR 1205983

8. Ambrosio, L., Caselles, V., Masnou, S., \& Morel, J.-M. Connected components of sets of finite perimeter and applications to image processing. J. Eur. Math. Soc. 3 (2001), 39-92. Zbl 0981.49024 MR 1812124

9. Ambrosio, L., Fusco, N., \& Pallara, D. Functions of Bounded Variation and Free Discontinuity Problems. Oxford Univ. Press (2000). Zbl 0957.49001 MR 1857292

10. Ambrosio, L., \& Paolini, E. Partial regularity for the quasi minimizers of perimeter. Ricerche Mat. 48 (1999), 167-186. Zbl 0943.49032 MR 1765683

11. Bellettini, G., Caselles, V., \& Novaga, M. The total variation flow in $\mathbb{R}^{N}$. J. Differential Equations 184 (2002), 475-525. Zbl 1036.35099 MR 1929886

12. Bellettini, G., Chermisi, M., \& Novaga, M. Crystalline curvature flow of planar networks. Interfaces Free Bound. 8 (2006), 481-521. Zbl 1103.74017| MR 2283923

13. Bombieri, E. Regularity theory for almost minimal currents. Arch. Ration. Mech. Anal. 78 (1982), 99130. Zbl 0485.49024 MR 0648941

14. Braides, A. Approximation of Free-Discontinuity Problems. Springer, Berlin (1998). Zbl 0909.49001 MR 1651773

15. Burago, Y. D., \& A. Zalgaller, V. Geometric Inequalities. Springer, New York (1988). Zbl 0633.53002 MR 0936419

16. Caraballo, D. G. A variational scheme for the evolution of polycrystals by curvature. Princeton Univ. Ph.D. thesis (1997) (Thesis advisor: Frederick J. Almgren, Jr.). MR 2695091

17. CARABALlo, D. G. 2-Dimensional flat curvature flow of crystals. Interfaces Free Bound. 7 (2005), 241254. Zbl pre02215422 MR 2171131 
18. Caraballo, D. G. Crystals and polycrystals in $\mathbb{R}^{n}$ : lower semicontinuity and existence. J. Geom. Anal. 18 (2008), 68-88. Zbl 1191.49011 MR 2365668

19. Caraballo, D. G. The triangle inequalities and lower semi-continuity of surface energy of partitions. Proc. Roy. Soc. Edinburgh Sect. A 139 (2009), 449-457. Zbl 1168.49040 MR 2506781

20. Chan, T. F., \& Esedoglu, S. Aspects of total variation regularized $L^{1}$ function approximation. SIAM J. Appl. Math. 65 (2005), 1817-1837. Zbl 1096.94004 MR 2177726

21. De Giorgi, E. Su una teoria generale della misura $(r-1)$-dimensionale in uno spazio ad $r$ dimensioni. Ann. Mat. Pura Appl. (4) 36 (1954), 191-212; reprinted in: Ennio De Giorgi: Selected Papers (L. Ambrosio et al., eds.), Springer (2006). Zbl 0055.28504 MR 0062214

22. De Giorgi, E. Nuovi teoremi relativi alle misure $(r-1)$-dimensionali in uno spazio ad $r$ dimensioni. Ricerche Mat. 4 (1955), 95-113; reprinted in: Ennio De Giorgi: Selected Papers (L. Ambrosio et al., eds.), Springer (2006). Zbl 0066.29903 MR 0074499

23. Evans, L. C., \& GARIEPY, R. F. Measure Theory and Fine Properties of Functions. CRC Press (1992). Zbl 0804.28001 MR 1158660

24. Federer, H. The Gauss-Green theorem. Trans. Amer. Math. Soc. 58 (1945), 44-76. Zbl 0060.14102 MR 0013786

25. Federer, H. Geometric Measure Theory. Springer (1969). Zbl 0176.00801 MR 0257325

26. Giusti, E. Minimal Surfaces and Functions of Bounded Variation. Birkhäuser, Boston (1984). Zbl 0545.49018 MR 0775682

27. Gurtin, M. E., Williams, W. O., \& Ziemer, W. P. Geometric measure theory and the axioms of continuum thermodynamics. Arch. Ration. Mech. Anal. 92 (1986), 1-22. Zbl 0599.73002 MR 0816619

28. Krantz, S. G., \& Parks, H. R. The Geometry of Domains in Space. Birkhäuser, Boston (1999). Zbl 0929.26001 MR 1730695

29. KrantZ, S. G., \& PARKs, H. R. Geometric Integration Theory. Birkhäuser, Boston (2008). Zbl 1149.28001 MR 2427002

30. Luckhaus, S., \& Sturzenhecker, T. Implicit time discretization for the mean curvature flow equation. Calc. Var. 3 (1995), 253-271. Zbl 0821.35003 MR 1386964

31. Mattila, P. Geometry of Sets and Measures in Euclidean Spaces: Fractals and Rectifiability. Cambridge Univ. Press (1995). Zbl 0819.28004 MR 1333890

32. Meyer, Y. Oscillating Patterns in Image Processing and Nonlinear Evolution Equations. Amer. Math. Soc. Univ. Lecture Ser. 22 (2001). Z Zbl 0987.35003 MR 1852741

33. Morgan, F. Geometric Measure Theory: A Beginner's Guide. 4th ed., Academic Press, New York (2009). Zbl 1179.49050 MR 2455580

34. Nikolova, M. Minimizers of cost-functions involving nonsmooth data-fidelity terms. SIAM J. Numer. Anal. 40 (2002), 965-994. Zbl 1018.49025 MR 1949401

35. Rudin, L., OSher, S., \& FATEMI, E. Nonlinear total variation based noise removal algorithms. Phys. D 60 (1992), 259-268. Zbl 0780.49028

36. Strong, D., \& Chan, T. F. Edge-preserving and scale-dependent properties of total variation regularization. Inverse Problems 19 (2003), S165-S187. Zbl 1043.94512 MR 2036526

37. TAmanini, I. Boundaries of Caccioppoli sets with Hölder-continuous normal vector. J. Reine Angew. Math. 334 (1982), 27-39. Zbl 0479.49028 MR 0667448

38. TAYlor, J. E. Some mathematical challenges in materials science. Bull. Amer. Math. Soc. 40 (2003), 69-87. Zbl 1158.74438 MR 1943134

39. Vixie, K. R., \& Esedoglu, S. Some properties of minimizers for the $L^{1}$ TV functional. Preprint.

40. YIP, N. K. Stochastic curvature driven flows. In: Stochastic Partial Differential Equations and Applications (Trento, 2002), Lecture Notes in Pure Appl. Math. 227, Dekker, New York (2002), 443-460. Zbl 0998.60096 MR 1919523 